\title{
Coordinated Control of SVC and TCSC for Voltage Profile Improvement employing Particle Swarm Optimization
}

\author{
Maulik Pandya $^{\# 1}$, Dr J G Jamanani ${ }^{* 2}$ \\ ${ }^{\#}$ Research Scholar, Department of Elect. Engg., KSV, Gandhinagar, Gujarat, India. \\ ${ }^{1}$ mcpandya_ee@ldrp.ac.in \\ *Asso. Prof. , Department of Elect. Engg., PDPU, Gandhinagar, Gujarat, India. \\ 2 jg.jamnani@sot.pdpu.ac.in
}

\begin{abstract}
Flexible AC transmission system (FACTS) devices are widely used in power system. They have characteristics to perform faster and can solve many problems in power system study. With regards to this, a particle swarm optimization (PSO) algorithm is applied so that to design the coordinated parameters of static VAR compensator (SVC) and Thyristor Controlled Series Capacitor (TCSC) is presented here. With behavioural nonlinearities of the power system, linear methods cannot be used to design coordinated parameters of controllers. Simulations has been done on IEEE (WSCC) 9 bus power system in MATLAB and its toolboxes. The results are compared and found that coordination of FACTS devices with each other confirm the efficiency of the proposed method for improving the voltage profile. When operation of TCSC is constrained by some limitation, adjustable SVC can supply auxiliary support to improve the overall performance.
\end{abstract}

Keyword - Static Var Compensator (SVC), Power System Stabilizer (PSS), Particle Swarm optimization (PSO), Coordination

\section{INTRODUCTION}

Flexible ac transmission system controllers have ability to extend the power transfer capability and also to enhance the stability within given limits, along with improvements in power system operation [3]. Voltage stability has been seen as a steady state problem involving static power flow studies for analysis. The voltage at various buses, the flow of active and reactive power, etc. keep on changing. It is understood that the FACTS controller will normally be equipped with higher order controllers such as power swing damping controller, Sub Synchronous Resonance (SSR) damping controller etc. Still to develop an insight, the FACTS controller is assumed to be equipped with simple PI controller only [1]. For improvement in operation of power system kind of interaction can occur amongst different types of FACTS controllers, which may include the interaction of stabilizers and High voltage DC (HVDC) controllers, too. It is categorized on the base of different frequency ranges. The term coordination haven't a meaning for centred control; instead, it understood that the tuning of the FACTS controllers will be done together for ensuring the promising, positive improvement of the overall control scheme. It is implied that the each controller depends chiefly on measure of topically useable quantity. It will act separately on connected FACTS devices.

The TCSC is an important component of FACTS (Flexible AC Transmission Systems). With the firing control of the thyristor, it can change its apparent reactance smoothly and rapidly. The TCSC is able to directly schedule power flow along desired paths and allow the system to operate closer to the line limits [9]. The SVC is a shunt compensation component. It is originally designed for voltage maintenance in power systems. Much as the TCSC, the SVC is also capable of flexible adjustment. In conventional methods, when performance of power system is normally linear, it is designed and engaged cautiously. In the occurrence of large perturbation, power system operating point changes considerably. However, in these conditions, nonlinearities of power system have substantial effects and linear methods cannot be capable of endure its stability [10]. Therefore, it is essential to consider the effect of nonlinearities of power system [9]. Recent years, intelligent optimization algorithms has been broadly used to design power system stabilizers. In this context, tabu search algorithm [6], genetic algorithm [7], simulated annealing, bacterial foraging algorithm (BFA) and small population-based PSO are applied as intelligent algorithms for one to one coordination of stabilizer in power system. In various literature many researchers proposed the coordination betwixt Power system stabilizer and FACTS controller for encompassing the dynamic performance of the power system. In [8], global tuning procedure for PSS and FACTS devices using a parameter forced no dimensional optimization algorithm is shown. The integral of squared error technique is used for global tuning of the stabilizers by considering the multi machine power system containing SVC and TCSC as well as PSS to manifest the efficient and hardiness of the proposed tuning procedure is discussed in [10]. 


\section{SVC \& TCSC}

According to IEEE definition, SVC is a shunt connected static var generator is a static var generator capable of stepless adjustment of reactive power over an unlimited range i.e. lagging or leading without any time delay.. In its basic form it comprises of fixed capacitor bank and switched reactor bank in parallel. A duet of reverse poled thyristors is connected in series with a fixed inductor to frame a Thyristor Controlled Reactor (TCR) module. When the thyristors are connected in series with a capacitor they will pattern Thyristor Switched Capacitor module. These will draw reactive power from the line and thereby regulating voltage, improve both steady-state and dynamic stability and reduce voltage flicker. Hence it will drastically improve the voltage profile.

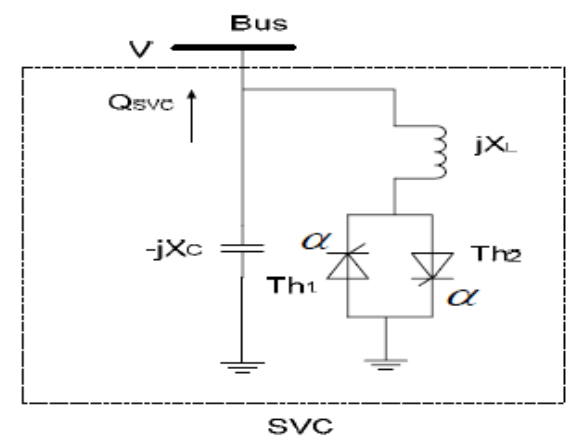

Fig. 1 SVC firing angle mode

The main chore of SVC is to endure the voltage of a specific bus by reactive power compensation which is normally done by adjusting the firing angle of the thyristors. In addition to this, Static VAR Compensator (SVC) is normally connected in parallel with line and main function is to modulate the voltage at a given bus by controlling its equivalent reactance. Generally there are two configurations of the SVC i.e. as per Fig. 1. The SVC can be modelled as a first order linear differential equation model. Basically, it is at the middle of a transmission line or sometimes at a load bus, too.

The basic configuration of the TCSC is shown in Fig.2. The system consists of a thyristor-controlled reactor (TCR), a parallel capacitor and a Metal Oxide Varistor (MOV). There are operating limits in the firing-angle, voltage protection level of the MOV and the harmonic current, dependent on the line current. Hence, the reactance range is also limited, depending on the current. The capability of the TCSC can be illustrated in terms of its reactance versus the line current.

TCSC acts as the inductive or capacitive compensator by modifying equivalent reactance $X_{\text {TCSC }}$ of transmission line [13]. Its value is controlled within certain capacitive reactance range while supplying damping to power systems. In the transient process, this controls line power flow by rapidly changing equivalent reactance. The TCSC provides powerful means of controlling and increasing power transfer level of a system by varying the apparent impedance of a specific transmission line. A TCSC can be utilized in a planned way for contingencies to enhance power system stability.

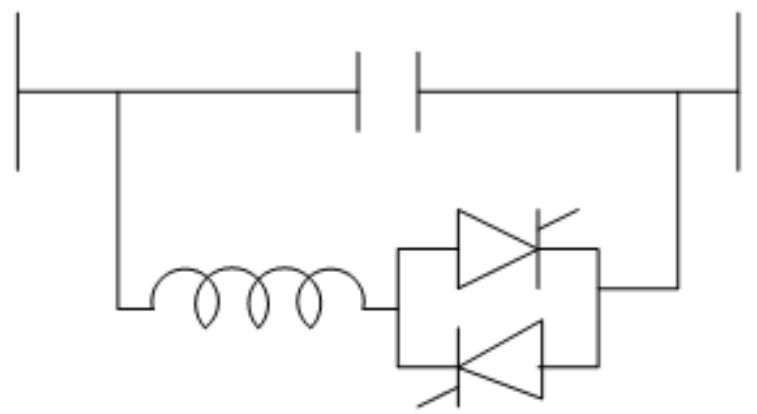

Fig. 2 Basic structure of TCSC 


\section{III.PARTICLE SWARM OPTIMIZATION}

\section{A. Basics of PSO}

Particle swarm optimization is a robust stochastic optimization technique based on the movement and intelligence of swarms. PSO applies the concept of social interaction to problem solving. It was developed in 1995 by James Kennedy (social-psychologist) and Russell Eberhart (electrical engineer) [4]. PSO has a pliant and finely adjusted mechanics to raise the global and local expedition abilities. It uses a number of agents (particles) that constitute a swarm moving around in the search space looking for the best solution. Each particle is treated as a point in a N-dimensional space which adjusts its "flying" according to its own flying experience as well as the flying experience of other particles. The particles in the optimization problem contribute their data with each other and run towards the best flight for detecting optimal result by number of iterations. In apiece iteration particles will have to update their velocities and positions by using the following equations:

where,

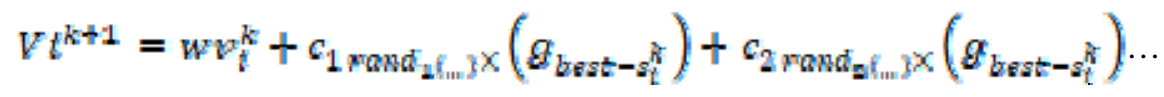

$\mathrm{V}_{\mathrm{i}}^{\mathrm{k}}=$ Velocity of agent I at iteration $\mathrm{k}$,

$\mathrm{W}=$ Weighting function,

$\mathrm{C}_{\mathrm{i}}=$ weighting factor,

rand $=$ uniformly distributed random number $\mathrm{b} / \mathrm{w} 0$ and 1 ,

${ }_{\mathrm{i}}^{\mathrm{k}}=$ current position of agent $\mathrm{I}$ at iteration $\mathrm{k}$,

$\mathrm{p}_{\text {best }}=$ personal best position of agent $\mathrm{I}$,

$\mathrm{g}_{\text {best }}=$ global best of the group.

Equation (2) is normally utilized in (1)

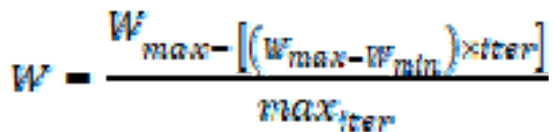

where,

$\mathrm{w}_{\max }, \mathrm{w}_{\min }=$ Max. \& Min. Inertia weight,

$\max _{\text {iter }}=$ maximum iteration number,

iter $=$ current iteration number.

The PSO iteration is carried out to obtain the smooth voltage profile according to the algorithm [4] of it, as shown in fig. (3). The optimization problem considered in this case is to minimize the cost. The objective function in this optimization problem is used as a fitness function in the PSO.

TABLE 1 PSO Parameters

\begin{tabular}{|c|c|}
\hline No. of Particles & 10 \\
\hline Max. Inertia weight & 0.9 \\
\hline Min. Inertia weight & 0.4 \\
\hline $\mathbf{C}_{\mathbf{1}}, \mathbf{C}_{\mathbf{2}}$ & $0.5,3.5$ \\
\hline No. of Iterations & 10 \\
\hline
\end{tabular}

\section{B. Why PSO?}

From an evolutionary point of view, the functioning of the PSO is wagerer with respect to GA [12] and then it is proved that PSO arrives at its terminal parameter values in very lesser number of generations than the GA. Apart from that it was found that several stabilizers like PSS, SVC, TCSC etc. are individually can improve the system stability. With compare to GA, PSO is gentle to implement and it comprises of very less parameters to adapt [11]. All particles in PSO are kept as members of the population through the course of the run. PSO is the only algorithm that does not implement the survival of the fittest. No crossover operation in PSO. In Evolutionary Programming (EP), poise between the global and local search should be managed through the strategy parameter while in PSO the poise will accomplish by the inertial weight factor (w). Apart from all, PSO has many variants like discrete PSO, MINLP PSO, Hybrid PSO etc. The conventional PSO has demerit of its short of convergence towards global optima. 


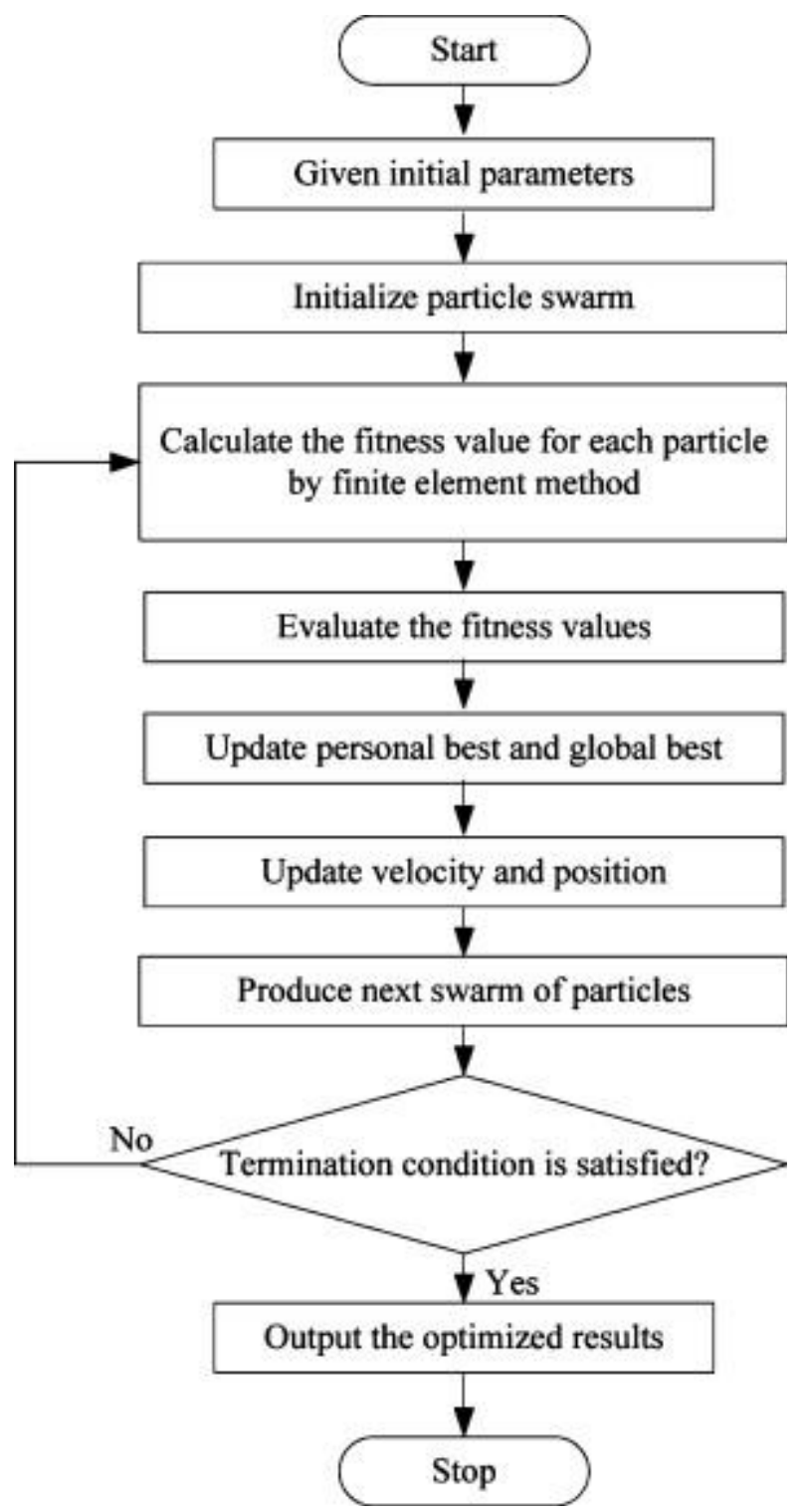

Fig. 3 Algorithm of PSO

\section{A. Power System Model}

\section{IV.PROBLEM FORMULATION}

To examine power system, the Western System Coordinated Council (WSCC) 3- machine, 9-bus system shown in Fig. 4 is depicted. Base MVA is considered as 100 MVA, and system frequency is taken as $60 \mathrm{~Hz}$. The system parameters is also given in Appendix. In Fig. 4 Bus numbers are shown as 1,2,3,..,9.

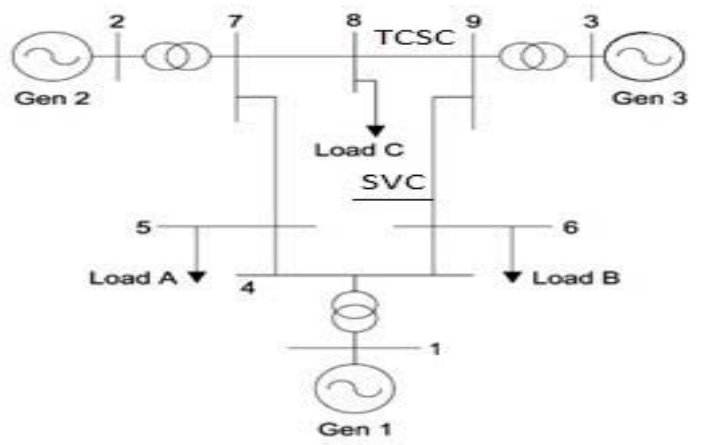

Fig. 4 WSCC, 9-Bus system 


\section{RESULT AND DISCUSSION}

In this study, PSO algorithm is put upon to navigate the parameters of SVC and TCSC controller coordinately by solving equation (1). In order to formalize the said method, the simulations are put through the multi machine system in MATLAB using its toolboxes. PSO [4] used here is based on finding the value of L \& C of SVC from which we get good and fine co-ordination. Here, PSO is used with some modification like change in PSO range \& number, change in selection criteria technique, control technique compare to Generalize PSO. Now the execution of the projected coordinated act is studied for a multi machine system as per Figure 4 with a SVC of 300 MVA installed at bus 6. Also, between transmission line 8-9, TCSC is connected.

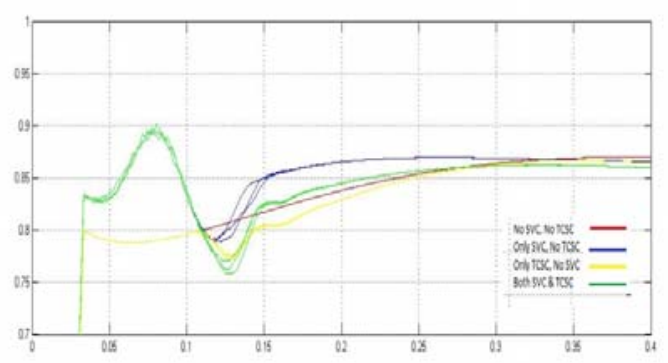

Fig. 5(a) Voltage AT Bus1

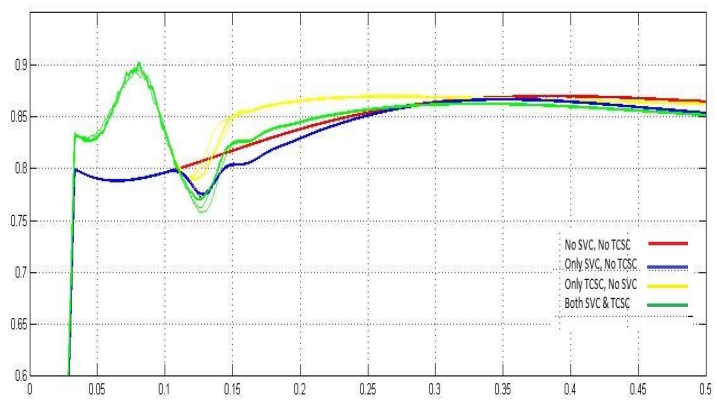

Fig. 5(b) Voltage at Bus 2

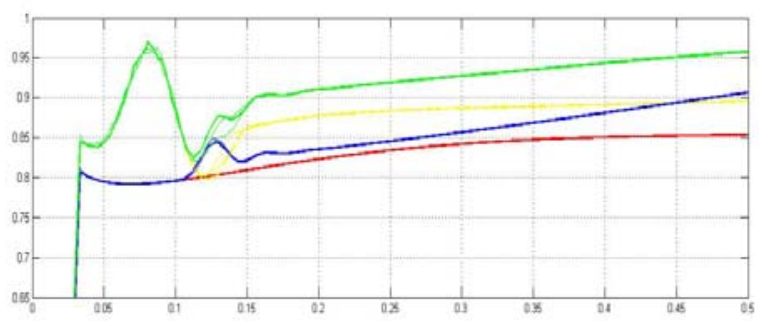

Fig. 5(c) Voltage at Bus 6

SVC initiation in the transmission line for bettering the voltage profile in the system is being carried. Parameters of PSO algorithm is as per Table 1. Results for voltage profile improvement at buses 1, 2 and 3 are tabulated in Table 2. Figure 5(a), (b) and (c) shows the simulation results for Bus 1, Bus 2 and Bus 6 voltages respectively. Dotted (green) line shows the effect of coordinated control of SVC and TCSC.

This is due to the fact that the installation of SVC has modified the overall reactance of the transmission line which subsequently ameliorate the voltage profile in the system. Also, TCSC generates reactive power and thus improving the Voltage Stability of the System. From the table, it is observed that once SVC and TCSC are installed in the system, voltage profiles of the bus for different loading conditions have been improved substantially. 
TABLE 2 Voltage Profile at various Buses

\begin{tabular}{|c|c|c|c|c|}
\hline Case & $\begin{array}{c}\text { No SVC, NO } \\
\text { TCSC }\end{array}$ & $\begin{array}{c}\text { Only SVC, } \\
\text { No TCSC }\end{array}$ & $\begin{array}{c}\text { No SVC, } \\
\text { Only TCSC }\end{array}$ & $\begin{array}{c}\text { All TCSC, SVC } \\
\text { connected } \\
\text { (Coordination) }\end{array}$ \\
\hline Bus1 Voltage & 0.86 & 0.88 & 0.87 & $\mathbf{0 . 9}$ \\
\hline Bus2 Voltage & 0.85 & 0.865 & 0.855 & $\mathbf{0 . 8 7}$ \\
\hline Bus3 Voltage & 0.855 & 0.86 & 0.855 & $\mathbf{0 . 8 7 5}$ \\
\hline Bus6 Voltage & 0.85 & 0.09 & 0.87 & $\mathbf{0 . 9 6}$ \\
\hline
\end{tabular}

\section{VI.CONCLUSION}

This paper gives a concise idea on effect of Coordination. Their individual contribution towards the improvement of voltage profile which has been tested on a 9-bus system. The combination of SVC and TCSC has been considered, as a multi type FACTS devices effect on the maintenance of the voltage profile. The proper tuning optimizes the parameters of the controllers. Also, results of simulation clearly validating that Voltage Profile is effectively improved with coordinated control technique.

\section{REFERENCES}

[1] I. Musirin, Nur D.M.Razdi, M.K.Idris "Voltage Profile Improvement using UPFC via Artificial Immune System," WSEAS transactions on Power System, ISSUE 4, Vol.-III, April 2008 , pp. 194-204.

[2] S. panda, N.P.Padhy, "Coordinated design of TCSC controller and PSS employing Particle Swarm optimization Technique" World Academy of science, Engineering \& Technology, pp. 427-435, April 2007.

[3] R. Narne, P.C.Panda, "PSS with multiple FACTS controller coordinated design and real time implentation using advanced adaptive PSO,” World Academy of science, Engineering \& Technology, Vol. VIII, . 137-147, Jan.-2014

[4] Y.D.Valle, G.K.Venayagamoorthy, S. mohagheghi, and R.G.Harley, "Particle Swarm Optimization:Basic concepts, variants \& applications in power systems,” IEEE transaction on evolutionary computation, vol. 12, No. 2 April 2008, pp. 171-195.

[5] Rui Min, Fei Xu, Fei Yuan, Zonghe Gao, " Coordinated Control of Multi-FACTS to Enhance the Dynamic Stability of the Power System ” Energy and Power Engineering, 2013, PP 1187-1191.

[6] Shareef, H.; Mohamed, A. "Coordinated design of unified power flow controller and power system stabilizer to enhance power system stability”, (POWERCON), 2012 IEEE International Conference on PP:1-6.

[7] Shayeghi, H., Shayanfar, H.A., Safari, A., Aghmasheh, R., "A robust PSSs design using PSO in a multi-machine environment," Energy Convers Manage, 2010,Vol. 51,

[8] No. 4, pp. 696-702.

[9] Xianzhang Lei, Edwin N. Lerch, Dusan Povh, Optimization and Coordination of Damping Controls for Improving System Dynamic Performance, IEEE Transaction on Power System., 16 (2001), pp. 473-480.

[10] S.K.Tso, j. Liang, Q.Y.Zeng ," Coordination of TCSC and SVC for Stability improvement of power systems, Proceedings of $4^{\text {th }}$ Int. conference on APSCOM-1997, Hong Kong..

[11] Xianzhang Lei, Edwin N. Lerch, Dusan Povh, "Optimization and co- ordination of Damping Controls for Improving System Dynamic Performance”, IEEE Trans. Power Systems, 16 (2001) 473 - 480.

[12] L.H.Hassan, Mahamoud Moghavveni, K.M.Muttaqi, "A Coordinated design of PSS and UPFC-based stabilizer using Genetic Algorithm”, IEEE transactions on Industry Applications, Vol. 50, No. 5, Oct. 2014, pp. 2957-2966

[13] S. panda, N.P.Padhy, "Comparison of particle swarm optimization and Genetic Algorithm for FACTS-based controller design" Applied soft computing, Vol-8, pp. 1418-1427, 2008.

[14] El Metwally M M, El Emary A A, El Bendary F M, et al. Optimal allocation of FACTS devices in power system using genetic algorithms, Power System Conference, 2008. MEPCON 2008. $12^{\text {th }}$ International Middle-East, IEEE, 2008: 1-4.

\section{APPENDIX}

\begin{tabular}{|c|c|c|c|}
\hline GENERATOR NO. & $\mathbf{1}$ & $\mathbf{2}$ & $\mathbf{3}$ \\
\hline RATED MVA & 247.5 & 192 & 128 \\
\hline KV & 16.5 & 18 & 13.8 \\
\hline Power Factor & 1 & 0.85 & 0.85 \\
\hline
\end{tabular}

